\title{
INFLUÊNCIA DA VASSOURA DE BRUXA NA FENOLOGIA DO CUPUAÇUZEIRO
}

\author{
Martha de Aguiar FALCÃO', Ronaldo Ribeiro de MORAIS', Charles Roland \\ CLEMENT $^{3}$
}

RESUMO - O cultivo do cupuaçuzeiro [Theobroma grandiflorum (Willd. ex Spreng.) Schum.] está em franca expansão na Amazônia brasileira. O principal fator limitante é a vassoura de bruxa, doença causada pela Crinipellis perniciosa (Stahel) Singer, cujo único controle econômico conhecido é a poda fitossanitária. A fenologia de dez cupuaçuzeiros em monocultivo, em solo Latossolo Amarelo distrófico adubado na região de Manaus, AM, foi avaliada de maio 1987 a dezembro 1990. O número de flores foi reduzido pela vassoura e pela poda, mas a duração e periodicidade da floração não foram muito afetadas. O número

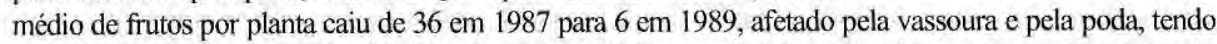
recuperado para 15 em 1990. A frutificação foi antecipada e sua duração reduzida em comparação ao estudo anterior, possivelmente devido à adubação e/ou ao genótipo estudado. $\mathrm{O}$ vingamento caiu de $1,4 \% \mathrm{em}$ 1987 para $0,5 \%$ em 1989 e aumentou para 1,8\% em 1990, sugerindo a recuperação das plantas. A brotação foliar e queda das folhas aumentaram marcadamente como resultado da influência da vassoura de bruxa em 1988 e foram reduzidas pela poda no período de 1989-90. Os fenofases năo foram muito afetados pela vassoura de bruxa embora o número de flores e frutos tenham sido.

Palavras chave: Theobroma grandiflorum, Crinipellis perniciosa, poda fitossanitária, floração, frutificação.

\section{Effect of Witch's Broom Disease on the Phenology of Cupuassu}

ABSTRACT - The cupuassu (Theobroma grandiflorum [Willd. ex Spreng.] Schum.) is expanding rapidly as a fruit crop in Brazilian Amazonia. The principal limiting factor is witch's broom disease, caused by Crinipellis perniciosa (Stahel) Singer, the only economic control for which is phytosanitary pruning. The phenology of 10 cupuassu plants grown in a fertilized monoculture on a nutrient poor Oxisol near Manaus, Amazonas, Brazil, was studied from May 1987 to December 1990. The number of flowers was reduced by the witch's broom and the pruning, but the duration and periodicity of flowering were not much affected. The mean number of fruits per tree fell from 36 in 1987 to 6 in 1989 as a result of the disease and its control, but recovered to 15 in 1990. Fruiting was slightly earlier and its duration was shorter than in a previous study, possibly due to the fertilization or the genotype studied. Fruit set fell from $1.4 \%$ in 1987 to $0.5 \%$ in 1989 and recovered to $1.8 \%$ in 1990 , suggesting recovery of the plants. Leaf and shoot flushing and leaf fall increased markedly as a result of the disease in late 1987 and 1988 and were markedly reduced by pruning in 1989-90. Phenophases were not much affected by witch's broom disease and its control, although the number of flowers and fruits were.

Key words: Theobroma grandiflorum, Crinipellis perniciosa, phytosanitary pruning, flowering, fruiting.

\section{INTRODUÇÃO}

A Amazônia é rica em espécies frutíferas de valor econômico. Uma das mais importantes hoje é o cupuaçuzeiro [Theobroma grandiflorum (Willd. ex Spreng.) Schum.], devido ao seu valor como fruta fresca ou industrializada. Seu mercado está expandindo tanto na região Norte como

1 Bolsista do $\mathrm{PCl}$ do Instituto Nacional de Pesquisas da Amazônia, modalidade DTI. Rua Silva Ramos, 758 - Centro, 69050-000 Manaus, AM, Brasil.

3 Centro de Pesquisas Agroflorestais da Amazônia Ocidental - CPAA/ EMBRAPA, Cx. Postal 399, 69011970 Manaus, AM, Brasil.

4 Instituto Nacional de Pesquisas da Amazônia - INPA, Cx. Postal 478, 69011-970 Manaus, AM, Brasil. 
no Brasil (Venturieri et al., 1993).

O gênero Theobroma possui 22 espécies, restritas à America Tropical; destas, nove são encontradas na Amazônia brasileira (Cuatrecasas, 1964). O cupuaçuzeiro é encontrado espontaneamente nas matas de terra firme e nas várzeas altas na parte sul e leste do Pará, estendendo-se também aos estados de Maranhão e Amazonas (Calzavara et al., 1984). As condições climáticas são variáveis nas áreas de ocorrência natural da espécie, com a temperatura média anual variando de 21,6 a $27,5^{\circ} \mathrm{C}$, precipitação anual de 1900 a $3100 \mathrm{~mm}$ e a umidade relativa do ar de 77 a $88 \%$ (Calzavara, 1987).

O plantio do cupuaçuzeiro tem aumentado na Amazônia, especialmente nos estados do Pará, Amazonas, Rondônia e Acre. No Amazonas, os municípios próximos a Manaus (Manacapuru, Presidente Figueiredo, Itacoatiara, Rio Preto da Eva, Iranduba) expandiram a produção de cupuaçuzeiro devido às perspectivas do mercado para sua polpa congelada no Brasil e no exterior. A polpa do cupuaçu é usada pelas indústrias alimentícias e caseiras para a fabricação de sucos, doces, geléias, compotas, pudins, balas, salames, licores, iogurtes, cremes e outros (Venturieri et al., 1993). As sementes do cupuaçu contém $48 \%$ de graxa branca e aromática, parecida com a manteiga de cacau (Le Cointe, 1934), que pode ser utilizada na fabricação de um chocolate, conhecido como cupulate, para distinguí-lo do chocolate do cacau (Venturieri et al., 1993).

Diversas pesquisas básicas sobre esta espécie têm dado subsídios para o manejo prático, entre as quais o estudo da fenologia
(Falcão \& Lleras, 1983). A fenologia trata das relações entre os fatores climáticos estacionais e os fenômenos biológicos periódicos das plantas, tais como a floração, a frutificação, mudança foliar, lançamentos foliares, ou seja, das épocas de crescimento e latência das plantas (Valle \& Ignacio, 1981). Tais estudos ajudam a definir o tempo dos tratos culturais e planejar a coleta de frutos. A floração e a frutificação do cupuaçuzeiro iniciam-se a partir do terceiro ano após o transplante ao campo e a planta entra em produção no quarto ano, quando se encontra em pleno desenvolvimento, chegando a produzir cerca de 12 frutos no quinto ano (Calzavara et al., 1984) e 17 frutos no sétimo ano (Falcão \& Lleras, 1983). A floração ocorre entre maio e setembro, que corresponde à estação mais seca do ano (Addison \& Tavares, 1951; Falcão \& Lleras, 1983). A duração da floração é variável entre árvores (de 59 a 108 dias) (Falcão \& Lleras, 1983). A safra se estende de dezembro a abril, que corresponde à época de maior pluviosidade (Addison \& Tavares, 1951; Falcão \& Lleras, 1983; Calzavara et al., 1984; Venturieri et al., 1993). A duração da frutificação é também variável, dependendo da duração da floração, do número de frutos, e de fatores exógenos à planta, como o estado nutricional e fitossanitário, polinização, e o manejo usado (Venturieri, 1994). Falcão \& Lleras (1983) observaram um vingamento de $0,48 \%$ em plantas sadias, sem adubação, perto de Manaus.

Um dos fatores limitantes à expansão do cultivo é a vassoura de bruxa, doença causada pelo fungo Crinipellis perniciosa (Stahel) Singer, que reduz significativamente a produção de frutos e 
cujo único controle é a poda fitossanitária (Calzavara et al., 1984; Venturieri et al., 1993; Stein et al., 1996). A Crinipellis perniciosa é causada por um fungo basidiomiceto encontrado em toda a região amazônica, tanto em plantas adultas como em mudas. Afeta tanto as partes vegetativas como as partes reprodutivas do cupuaçuzeiro (Venturieri et al., 1993). Os ramos apresentam um crescimento anormal, com brotação exagerada das gemas laterais e em seguida secam. Os frutos secam, ficando de cor escura e caem; algumas vezes ficam nas árvores e se mumificam. O ciclo da doença depende das condições climáticas e do manejo do plantio; vassouras velhas reproduzem inóculo com maior intensidade na época chuvosa (Venturieri et al., 1993; Stein et al., 1996). A vassoura de bruxa reduz significativamente a produção econômica e, em casos extremos, elimina a produção, chegando a matar as plantas. A única forma econômica de eliminar a vassoura de bruxa é a poda fitosssanitária, praticada uma vez por ano. Esta poda consiste na eliminação de galhos e frutos infectados e sua retirada do plantio, devendo os mesmos serem enterrados ou queimados (Calzavara et al., 1984). É de se supor que a doença e seu controle possam afetar a fenologia do cupuaçuzeiro mas este efeito não tem sido relatado até o presente.

O objetivo original do presente estudo foi acompanhar a fenologia de cupuaçuzeiro em monocultivo, pois estudos anteriores (Addison \& Tavares, 1951; Falcão \& Lleras, 1983) foram sobre plantas isoladas. No primeiro ano de observação o plantio ficou infestado com a vassoura de bruxa e, por esse motivo, avaliou-se também o efeito da vassoura de bruxa e da poda fitossanitária na fenologia e na produção de frutos de cupuaçuzeiro nestas condições.

\section{MATERIAL E MÉTODOS}

Foram escolhidas dez árvores ao acaso num plantio homogêneo de cupuaçuzeiro com quatro anos de idade, na Fazenda NAF6, km 14, Rodovia BR 174 (Manaus - Caracarai). O formato dos frutos das árvores estudadas é redondo, seguindo a classificação de Calzavara (1970). O plantio foi instalado numa área de 0,5 ha de uma capoeira de dez anos, com espaçamento de $3 \times 3 \mathrm{~m}$ e adubação na cova com esterco de galinha (3:I v:v solo:esterco). A adubação de cobertura foi feita uma vez por ano com $1 \mathrm{~kg}$ da fórmula 4-16-8 de N, $\mathrm{P}_{2} \mathrm{O}_{5}$ e $\mathrm{K}_{2} 0$. O solo é um latossolo Amarelo, álico, textura média.

Ribeiro (1976) define o clima de Manaus como "Afi", no esquema de Köppen, com médias anuais de 2418 $\mathrm{mm}$ de precipitação; $26,7^{\circ} \mathrm{C}$ temperatura e $87,5 \%$ de umidade relativa (CPGEINPA, com. pess., 1997). Os dados climáticos (Fig. 1A) foram obtidos da estação do Ministério da Agricultura em Manaus, a $30 \mathrm{~km}$ do plantio.

As observações iniciaram-se no $1^{\circ}$ dia de maio de 1987 e foram repetidas semanalmente até 31 de dezembro de 1990. Em cada árvore foram escolhidos três galhos ao acaso, nos quais se fez contagem de flores, frutos e brotos foliares, conforme a metodologia de Falcão \& Lleras (1983). As folhas caidas também foram contadas semanalmente. Os dados de brotos foliares e folhas caidas de setembro a dezembro 1990 foram perdidos.

No final de 1987 verificou-se a ocorrência da vassoura de bruxa, a qual foi 
controlada com uma poda fitossanitária em 1988, cortando-se os galhos afetados e retirando-os do local para serem queimados, como recomendado por Calzavara et al. (1984). Nos anos subsequentes (1989 - 1990), a poda foi feita duas vêzes ao ano porque observou-se que uma poda fitossanitária não foi suficiente para controlar a infestação.

Os insetos visitantes foram capturados entre as 5:00 e 10:00 horas e entre 18:00 e 20:00 horas na época da máxima floração. Foram fixados e montados para posterior identificação.

\section{RESULTADOS \& DISCUSSÃO}

\section{Floração}

A floração ocorreu entre abril e janeiro, com variação no início e no fim de ano a ano, e o pico da floração ocorreu em agosto e setembro (Fig. 1B). A fenologia está relacionada com os fatores climáticos (compare a Fig. $1 \mathrm{~A} \mathrm{e}$ 1B), com a floração ocorrendo principalmente na estação seca, como observado por Addison \& Tavares (1951) e Falcão \& Lleras (1983).

A floração diminuiu consideravelmente em 1989 e 1990 (Tab. 1) sob o efeito da vassoura de bruxa e da poda fitossanitária. Em 1987 e 1988 foram produzidas mais de 2000 flores por planta quando a vassoura de bruxa estava se instalando e a poda fitossanitária era menos intensa. Em comparação, em 1989 e 1990 foram produzidas cerca de 1000 flores por planta quando a vassoura estava sendo controlada por uma poda mais intensa.

\section{Frutificação}

A frutificação iniciou-se na estação seca, com o vingamento dos frutos. A maturação dos frutos começou no inicio das chuvas (novembro) e estendeu-se até janeiro, raramente abril (Fig. 1B). Estas safras foram mais precoces e de duração menor do que as observadas por Falcão \& Lleras (1983), possivelmente por serem genótipos diferentes, pelo fato das plantas serem mais jovens e terem sido adubadas, ou pela ataque da vassoura de bruxa. Existiu grande heterogeneidade na duração da frutificação das árvores estudadas, fenômeno já observado por Falcão \& Lleras (1983).

A produção de frutos no primeiro ano foi acima da média encontrada na literatura (Tab. 1), sendo maior que os 17 frutos/planta observados por Falcão \& Lleras (1983) e os 10 a 20 frutos/planta esperados por Calzavara et al. (1984) em plantas com esta idade, provavelmente devido à adubação. No segundo e terceiro ano, a produção caiu marcadamente, provavelmente como consequência da vassoura de bruxa e da poda fitossanitária usada para controlá-la. A partir do quarto ano, a produção começou a recuperar-se, embora não chegasse ao observado no primeiro ano. Esta recuperação na produção foi similar à observada em cacau (Theobroma $\mathrm{ca}$ cao) após a instituição da poda como prática sistemática (Mendes et al., 1997). A recuperação das plantas é mais clara quando a porcentagem de vingamento é examinada (Tab. 1), pois esta porcentagem atingiu o patamar original no quarto ano, sugerindo que as plantas estavam sadias, embora fisicamente menores devido à poda fitossanitária. 

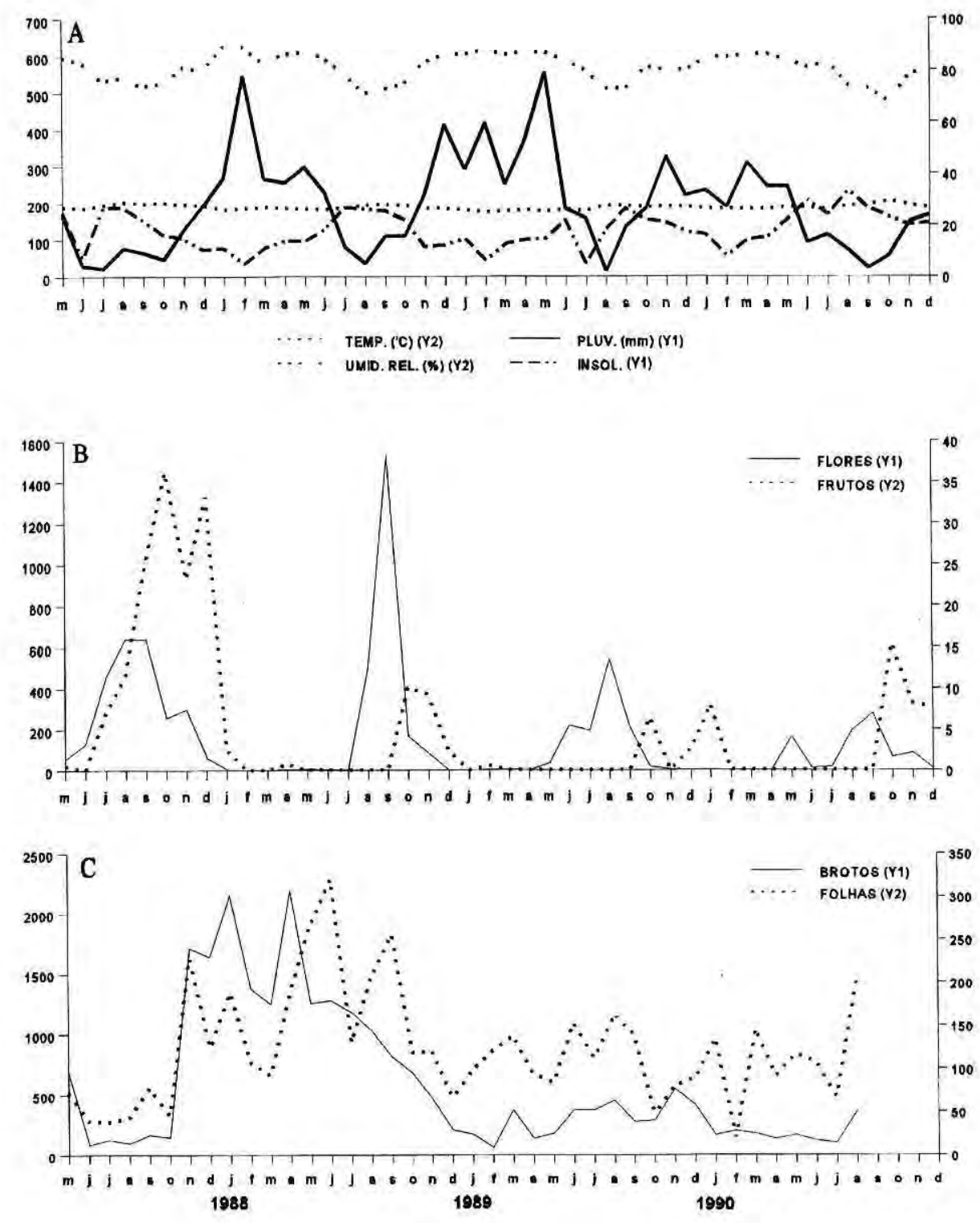

Figura 1. A. Dados climáticos de Manaus, Amazonas, Brasil, a $30 \mathrm{Km}$ do local das observações. B. Números de flores e frutos de cupuaçuzeiro observados mensalmente durante o período de maio 1987 a dezembro 1990. C. Número de brotações foliares e folhas caidas de cupuaçuzeiro observados mensalmente durante o período. 
Tabela 1. Médias do total de frutos e flores de cupuaçu produzidas por planta durante o período de observação, e a porcentagem de vigamento anual estimada.

\begin{tabular}{lcccc}
\hline & 1987 & 1988 & 1989 & 1990 \\
\hline Total de flores & 2532 & 2289 & 1217 & 804 \\
Total de frutos & 36 & 10 & 6 & 15 \\
\% vingamento & 1,42 & 0,44 & 0,49 & 1,86 \\
\hline
\end{tabular}

\section{Mudança foliar}

A queda das folhas e a brotação foliar ocorreram, lentamente, ao longo do ano inteiro, com claro efeito da vassoura de bruxa ao longo de 1988 (Fig. 1C). Falcão \& Lleras (1983) observaram que a queda das folhas ocorreu antes da floração e durante a frutificação. É comum em plantas que regiões em crescimento carreiam os nutrientes de regiões mais velhas, induzindo a senescência, sendo que no caso das folhas a consequência é a queda das mesmas. Esta sequência de queda das folhas começou a ser evidente a partir do terceiro ano, neste estudo, quando o efeito da vassoura de bruxa começou a diminuir. A priori, esperava-se um pico de brotação foliar após a frutificação e antes da próxima floração, quando as plantas estavam recompondo sua estrutura fotossintética. No entanto, não se observou uma relação consistente entre os picos de brotação foliar e a floração ou a frutificação.

\section{Insetos visitantes}

Os insetos visitantes coletados foram os mesmos encontrados por Falcão \& Lleras (1983). As abelhas mais freqüentes foram: Frieseomelitta silvestrii faceta Moure, Paratrigona impuctata Ducke e Apis mellifera adansoni.L. As formigas, especialmente as do gênero Camponotus, aumentaram em abundância consideravelmente em relação às observações realizadas por Falcão\& Lleras (1983). As formigas do gênero Cromatogaster apresentavam relações simbióticas com pulgões.

\section{CONCLUSÕES}

A floração e a frutificação aconteceu uma vez por ano. A vassoura de bruxa e a poda fitossanitária usada para controlá-la afetaram a fenologia e a produção de frutos. O número de flores caiu marcadamente com a evolução de $C$. perniciosa e seu controle, mas a periodicidade e duração da floração não mudaram. O número de frutos também caiu marcadamente, mas começou a recuperar antes do final das observações. A porcentagem de vingamento mostrou que as plantas provavelmente estavam se recuperando do ataque de $C$. perniciosa, provando a eficácia da poda fitossanitária em cupuaçuzeiro.

\section{AGRADECIMENTOS}

A primeira autora agradece ao Conselho Nacional de Desenvolvimento Científico e Tecnológico (CNPq) e ao Instituto Nacional de Pesquisas da Amazônia (INPA) pela bolsa\DTI concedida. Os autores agradecem aos Dr. Norman Penny, João Camargo, Jesus S. Moura, Warwick E. Kerr, e Francisco J. Aguilera Peralta pela identificação dos insetos visitantes, aos Drs. Eduardo Lleras e Sidney A. do Nascimento Ferreira pela revisão do manuscrito e as sugestões oferecidas, a professôra Maria 
Bernadete Bonini Alves pela revisão do texto em português, e a dois referees anônimos da Acta Amazonica pelas criticas e sugestões oferecidas.

\section{Bibliografia citada}

Addison, G.O.; Tavares, R.M. 1951. Observações sobre as espécies do gênero Theobroma que ocorrem na Amazônia. Boletim Técnico do Instituto Agronômico do Norte, 25: 1-20.

Calzavara, B.B.G. 1970. Fruteiras: abieiro, abricozeiro, bacurizeiro, biribazeiro, cupuaçuzeiro. Série Culturas da Amazônia, Instituto de Pesquisas e Experimentação Agropecuária do Norte (IPEAN), Belém. pp. 45-84.

Calzavara, B.B.G. 1987. Recomendações básicas: Cupuaçuzeiro. Centro de Pesquisas Agropecuárias do Trópico Úmido (CPATU- EMBRAPA), Belém.

Calzavara, B.B.G.; Müller, C.H.; Kahwage, O.N.C. 1984. Fruticultura Tropical: O cupuaçuzeiro - cultivo, beneficiamento e utilização do fruto. Centro de Pesquisas Agropecuárias do Trópico Úmido (CPATU/EMBRAPA), Belém.

Cuatrecasas, J.R. 1964. Cacao and its allies: a taxonomic revision of the genus Theobroma. Contrib. U.S. Nat. Herb., 35: 379-614.

Falcão, M.A.; Lleras, E. 1983. Aspectos fenológicos, ecológicos e de produtividade do cupuaçu (Theobroma grandiflorum (Willd. ex Spreng.) Schum.). Acta Amazonica, 13: 725-735.

Galvão, E.U.P.; Gomes, O.N.R.; Nogueira, O.L.; Stein, B.L.R.; Müller, C.H.; Nunes, L.M.A. 1996. Efeito da poda no controle da vassoura de bruxa em cupuaçuzeiro com e sem sombreamento. Relatório Anual 1995/ 1996. Centro de Pesquisas Agropecuárias do Trópico Úmido (CPATU-EMBRAPA), Belém. pp. 193-194.
Le Cointe, P. 1934. Arvores e plantas úteis da Amazônia. Series Amazônia Brasileira, 3. Livraria Clássica, Belém. p. 145-146.

Mendes, F.A.T.; Oliveira, C.P.; Silva, I.F,; Almeida, J.C.T.; Carvalho, M.M.; Teixeira, C.D.B.; Zacchi, A.H. 1997. Recuperação agro-econômica de áreas cacaueiras altamente infectadas por vassoura de bruxa (Crinipellis perniciosa) na Amazônia Brasileira. Boletim Técnico da CEPLAC, 14: 11-16.

Ribeiro, M.N.G. 1976. Aspectos climatológicos de Manaus. Acta Amazonica, 6(2): 229-233.

Stein, B.L.R,; Endo, T.; Albuquerque, C.E.; Conceição, O.E.H.; Gomes, O.N.R.; Müller, C.H.; Nascimento, M.R.; Nunes, L.M.A. 1996. Biologia e fisiologia de Crinipellis perniciosa do cupuaçuzeiro em relação à fitopatologia. Relatório Anual 1995/1996. Centro de Pesquisas Agropecuárias do Trópico Úmido (CPATUEMBRAPA), Belém. pp. 61-62.

Valle, A; Ignacio, J.D. 1981. Fenologia. Universidad Nacional de Colombia, Departamento de Recursos Forestales, Medellin.

Venturieri, G.A.; com contribuições de RonchiTelles, B.; Ferraz, I.D.K.; Lourde, M.; Hamada, N. 1993. Cupuaçu: a espécie, sua cultura, usos, e processamento. Clube do Cupu, Belém.

Venturieri, G.A. 1994. Floral biology of cupuaçu (Theobroma grandiflorum (Willdenow ex Sprengel) Schuman). Tesis de PhD, Department of Agricultural Botany, University of Reading, Reading, Inglaterra. 